\title{
Heterotaxy-spectrum heart defects in Zic3 hypomorphic mice
}

\author{
Allison M. Haaning ${ }^{1}$, Malgorzata E. Quinn ${ }^{1}$ and Stephanie M. Ware
}

BACKGROUND: Mutations in Zinc Finger Protein of the Cerebellum 3 (ZIC3) cause X-linked heterotaxy and isolated cardiovascular malformations. Recent data suggest a potential cell-autonomous role for Zic3 in myocardium via regulation of Nppa and Tbx5. We sought to develop a hypomorphic Zic3 mouse to model human heterotaxy and investigate developmental mechanisms underlying variability in cardiac phenotypes.

METHODS: Zic3 hypomorphic mice were created by targeted insertion of a neomycin cassette and investigated by gross, histologic, and molecular methods.

RESULTS: Low-level Zic3 expression is sufficient for partial rescue of viability as compared with Zic3 null mice. Concordance of early left-right molecular marker abnormalities and later anatomic abnormalities suggests that the primary effect of Zic3 in heart development occurs during left-right patterning. Cardiac-specific gene expression of Nppa (atrial natriuretic factor) and Tbx5 marked the proper morphological locations in the heart regardless of looping abnormalities.

CONCLUSION: Zic3 hypomorphic mice are useful models to investigate the variable cardiac defects resulting from a single genetic defect. Low-level Zic3 expression rescues the left pulmonary isomerism identified in Zic3 null embryos. Our data do not support a direct role for Zic3 in the myocardium via regulation of Nppa and Tbx5 and suggest that the primary effect of Zic3 on cardiac development occurs during left-right patterning.

P atterning of the left-right axis is an event in early vertebrate development that is necessary for proper organ asymmetry. Signaling pathways involved in left-right patterning are conserved in vertebrates, and perturbation of genes encoding proteins in these pathways leads to abnormal organ patterning and arrangement known as situs ambiguus or heterotaxy. Mutations in Zinc Finger Protein of the Cerebellum 3 (ZIC3) were the first genetic causes of heterotaxy identified in humans $(1,2)$, accounting for about $1 \%$ of sporadic and $75 \%$ of X-linked familial heterotaxy cases (3). ZIC3 is a member of the ZIC family of transcription factors, which are related to GLI proteins that mediate hedgehog signaling, a conserved developmental pathway important for left-right patterning (4). ZIC3 is able to bind and activate transcription at the GLI binding site (5-8), and we recently demonstrated that ZIC3 converts GLI3 from repressor to activator in vitro (9). In addition, we identified that Zic3 regulates limb digit number via its modifying effect on Gli3 and Shh expression levels. It has been surmised that ZIC3 may similarly affect left-right signaling through its possible involvement in Shh signaling $(10,11)$. Zic3 has also been shown to affect a conserved transforming growth factor $\beta$-signaling pathway important for left-right signaling via interaction with the ligand nodal. A genetic interaction is demonstrated by a more severe phenotype in Zic3/Nodal compound mutants. In addition, reduced expression of a Nodal enhancer-driven $\beta$-galactosidase transgene at the node in Zic3 null mice (12) and abnormal Nodal expression in Zic3 null mice (11) indicate disruption of this critical pathway by loss of function of Zic3. Proper left-right patterning also requires an intact midline, and Zic3 null mice exhibit a range of midline defects such as disrupted notochord formation, incomplete neural tube closure, vertebral malformations, and bent tails $(11,13)$. The exact mechanism by which Zic3 patterns the left-right axis remains unknown, but its role in left-right patterning is clearly essential and is conserved in human, mouse, zebrafish, and frog $(1,3,10,11,13-15)$.

Isolated cardiovascular malformations have been observed in patients with ZIC3 mutations (3,16-19), indicating that there may be a requirement for ZIC3 specifically in the heart separate from its role in left-right patterning for proper cardiovascular development. Recently, ZIC3 was shown to bind to serum response factor and synergistically coactivate important cardiac genes including $\mathrm{Nppa}$ (encoding atrial natriuretic factor), Tbx5, and Nkx2.5 in vitro (20). In that study, Zic3 was shown to be expressed in the developing mouse heart by reverse-transcriptase PCR (RT-PCR); however, the question of whether Zic3 is expressed at physiological levels in the developing heart has not been resolved because other studies show no detectable Zic3 gene expression during cardiogenesis by whole mount in situ hybridization (WISH) $(5,11)$. Thus, a potential role for Zic3 in the cardiac compartment remains controversial. A wide phenotypic variety of laterality-spectrum heart defects, such as transposition of the great arteries (TGA), atrial isomerism, and atrioventricular septal defects, have been observed in Zic3 null mice (11-14,20); however, because of early embryonic lethality secondary to gastrulation defects (21), it is difficult to collect adequate numbers of embryos with abnormal heart development to study laterality-spectrum 
heart defects. Here, we present Zic3 hypomorphic mice that have low early embryonic lethality and high penetrance of laterality-spectrum heart defects as a model to better understand the etiology of cardiovascular malformations.

\section{RESULTS}

\section{Analysis of Zic3 Expression}

Targeted insertion of a neomycin cassette into intron 1 of murine Zic3 (Figure 1a), which is located on the X chromosome,

\section{a}

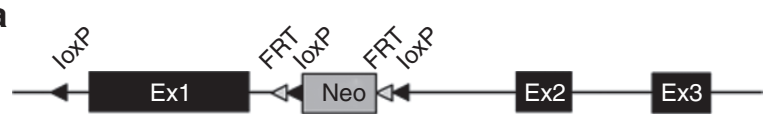

b

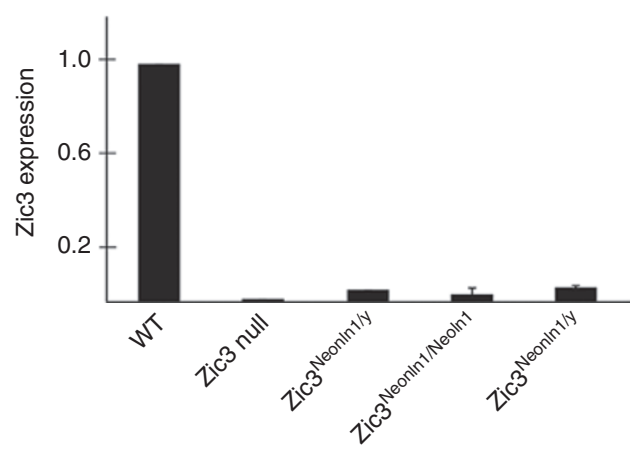

Figure 1. Disruption of intron 1 of Zic3 results in a hypomorphic Zic $3^{\text {Neol1 }}$ allele. (a) A neomycin cassette was incorporated into intron 1 of mouse Zic3 by targeted insertion. (b) The resulting Zic $3^{\text {Neoln1 }}$ allele exhibited reduced Zic3 expression by quantitative PCR (qPCR). Each bar in the (b) qPCR graph represents Zic3 expression level in one embryo of the indicated genotype. Null embryos are hemizygous if male (Zic $3^{\text {Neoln1/y) }}$ and homozygous if female (Zic3 $\left.{ }^{\text {Neoln1/Neoln1 }}\right)$. results in a hypomorphic allele, Zic $3^{\text {NeoIn } 1}$. Embryos containing only the Zic $3^{\text {Neorn } 1}$ allele (Zic $3^{\text {NeoIn1/NeoIn } 1}$ or Zic $3^{\text {Neorn1/y }}$ genotypes), which will hereafter be referred to as $Z i c 3$ hypomorphs, were shown to have a universal reduction of Zic3 expression by realtime RT-PCR (qPCR) (Figure 1b). The mean Zic3 expression level in embryonic day (E) 12.5 Zic3 hypomorphs was $4.7 \%$ compared with wild-type (WT) embryos ( $n=3$ for each genotype) by qPCR. These low levels of Zic3 expression were not able to be detected by WISH at E9.5 and E10.5 (Figure 2a,b and data not shown).

Zic3 expression has been detected in E10.5 hearts by qPCR and RT-PCR in previous studies, $(5,20)$ and recent work suggests a possible novel role in the ventricular myocardium via regulation of atrial natriuretic factor expression (20); however, its expression has not been detected in the heart at any embryonic stage by WISH (12). Previously, we described the generation of Zic3-LacZ-BAC reporter transgenic mice for more sensitive detection of Zic3 expression during development, (9) and we therefore sought to determine whether reporter gene expression was detectable in the cardiac compartment in vivo. Eight Zic3-LacZ-BAC transgenic lines were analyzed, and all lines had similar expression patterns from E6.5 to E12.5, which were also comparable with Zic3 WISH expression patterns from E6.5 to E12.5. At least three lines were analyzed at each of the following stages: E6.5, E8, E8.5, E9.5, E10.5, E11.5, and E12.5. Embryos were embedded and sectioned in order to analyze cardiac expression. Zic3 expression was not detected in the heart in any Zic3-LacZ-BAC line during embryonic development. A representative E12.5 Zic3-LacZ-BAC embryo is shown (Figure 2c), exhibiting no expression of $\beta$-galactosidase in the whole (Figure 2d) or sectioned (Figure 2e) heart.
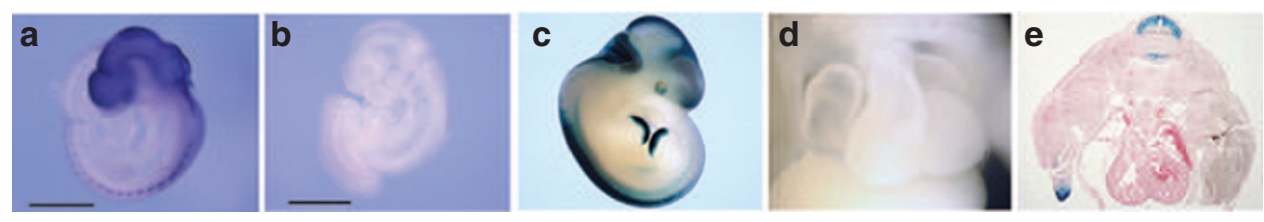

Figure 2. Analysis of Zic3 expression in the mouse embryo. Zic3 expression is (a) visualized in the wild-type (WT) embryo by whole mount in situ hybridization (WISH) and (b) not detectable by WISH in Neo embryos. Analysis of Zic3 expression using Zic3-LacZ-BAC transgenic mice (c) revealed no expression in the heart either (d) grossly or (e) histologically. Neo: Zic $3^{\text {Neoln1/Neoln1 }}$ or Zic3 ${ }^{\text {Neoln1/y. }}$. Scale bars in $\mathbf{a}$ and $\mathbf{b}=0.5 \mathrm{~mm}$.
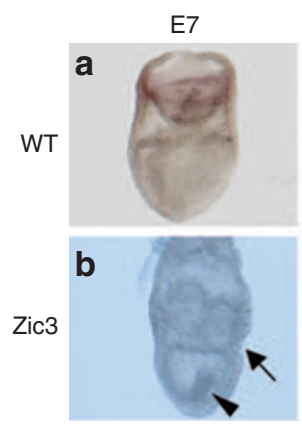
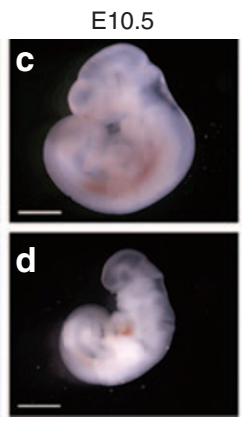
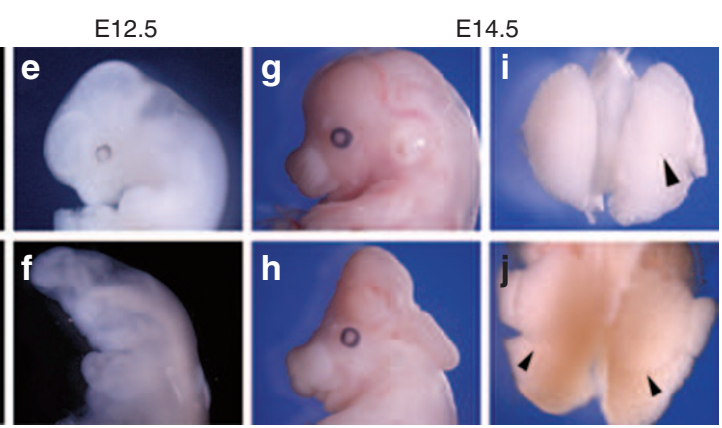

Figure 3. Representative phenotypes of Zic3 hypomorphs at different stages. Gastrulation abnormalities were observed in (b) some hypomorphic embryos (arrow, arrowhead; compared with wild-type (WT) embryo in (a)). Growth retardation was common in hypomorphs, compared with WT siblings ((c) WT; (d) hypomorph). (f,h) Neural tube defects were observed in many hypomorphs (compared with WT in e,g). Laterality defects such as right isomerism occurred frequently, indicated here by bilateral, multilobed lungs (j, arrowheads); compared with WT (i). Scale bars $=0.5 \mathrm{~mm}$. 


\section{Articles $\mid$ Haaning et al.}

Table 1. Analysis of genotype and phenotype at different developmental stages

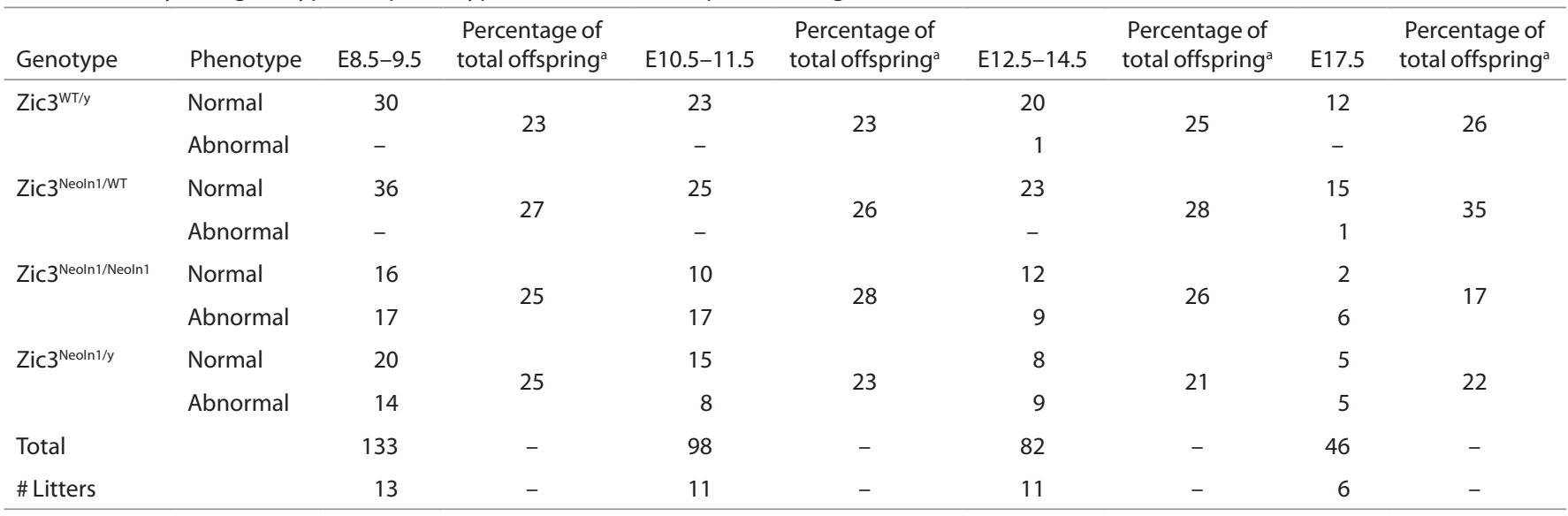

aTheoretical frequencies are 25\% for each genotype at all stages.
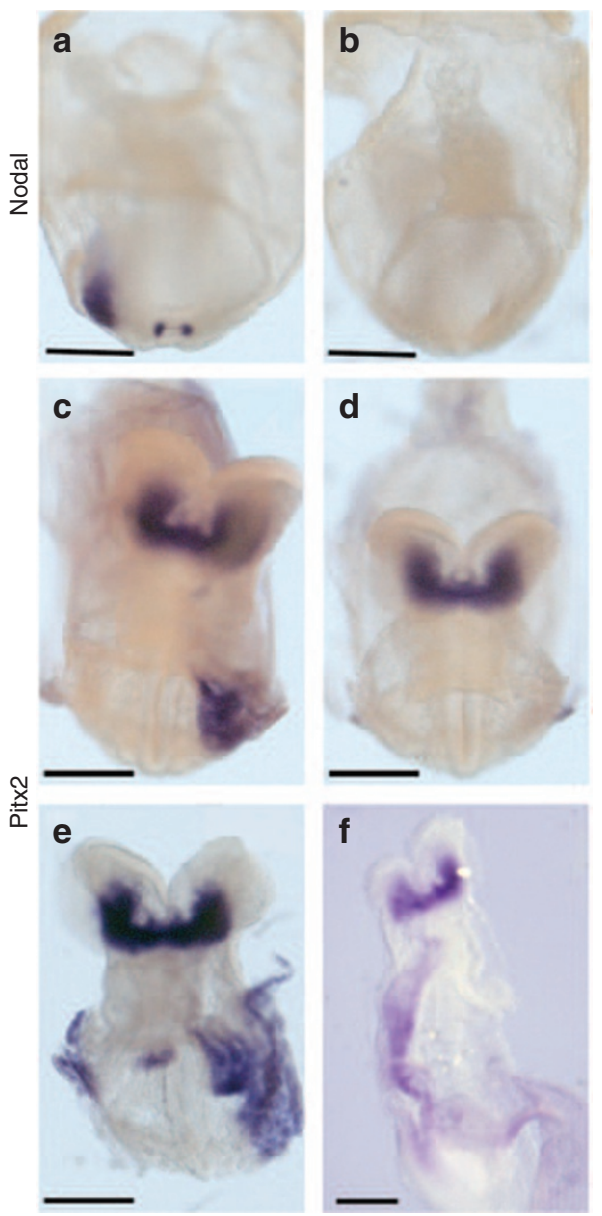

g

WT

4-6 som

Zic3 Neo

4-6 som

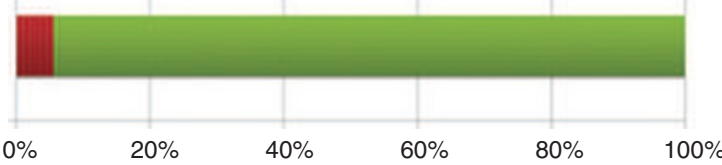

h

WT

4-6 som
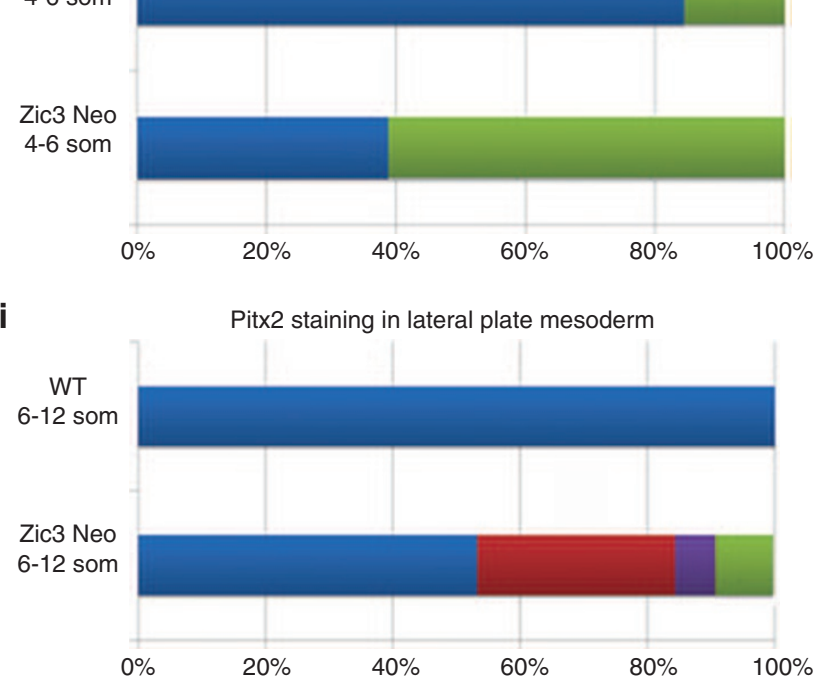

Figure 4. Abnormal left-right marker expression in Zic3 hypomorphs. WISH using a nodal riboprobe demonstrates bilateral, perinodal, and left lateral plate mesoderm (LPM) staining in (a) four-somite stage wild-type (WT) embryo compared with (b) four-somite stage Zic3 hypomorphic embryo with absent staining. (c) Left LPM Pitx2 expression in WT 10-somite stage embryo. Variable Pitx2 staining patterns in 8- to 10-somite Zic3 hypomorphs: (d) absent, (e) bilateral, and (f) right LPM. (g) Nodal staining patterns observed around the node in WTs and Zic3 hypomorphs (WT $n=13$, hypomorph $n=18$ ). Blue color represents left > right staining of node; red color represents bilaterally equal expression at the node; and green represents absent staining at the node. (h) Nodal staining patterns observed in LPM of WTs and Zic3 hypomorphs (WT $n=13$, hypomorph $n=18$ ). Blue color represents left LPM staining; green represents absent LPM staining. (i) Pitx2 staining patterns observed in LPM of WTs and Zic3 hypomorphs (WT $n=29$; hypomorph $n=45$ ). Blue color represents left LPM staining; green represents absent LPM staining; red represents bilateral LPM staining; and purple represents right LPM staining. Scale bars $=100 \mu \mathrm{m}$. 
Phenotypic Analysis of Zic3 Hypomorphs

Reduced expression of Zic3 led to phenotypes in embryonic and adult mice that were similar to those seen in Zic3 null mice $(11,21)$. Like Zic3 null mice, Zic3 hypomorphs exhibited gastrulation defects, delayed growth, craniofacial defects, neural tube closure defects, and a variety of laterality defects (Figure 3). Zic3 hypomorphs exhibited very modest embryonic or fetal lethality (Table 1), making them a useful model for studying complex cardiovascular malformations during and after cardiac looping. In addition, the Zic3 hypomorphs show a decreased rate of phenotypic abnormalities at early stages as compared with Zic3 nulls. Fifty-one percent of hypomorph females show phenotypic abnormalities before E9.5 (Table 1) compared with 81\% of null females at the same stage (21). Males show a similar difference between hypomorphs (41\% phenotypically abnormal) and nulls (61\% abnormal (21)).

The most common defects observed in gastrulating Zic3 hypomorphs were constriction between embryonic and extraembryonic tissue and accumulation of tissue in the proamniotic cavity (Figure 3b; arrow), which are the same defects seen in Zic3 nulls. In Zic3 nulls, the tissue in the amniotic cavity (Figure 3b; arrowhead) was previously shown to be mesoderm by WISH, using the mesodermal markers T-brachyury, Fgf8, and Wnt3a (21). This tissue was also shown to be mesoderm in Zic3 hypomorphs by Fgf8 WISH (data not shown). Accumulation of mesoderm tissue in the proamniotic cavity shows that this tissue is properly specified but that it is unable to incorporate into a definitive germ layer, which is required for completion of gastrulation and proper embryonic patterning $(22,23)$. Despite the appearance of gastrulation defects, most Zic3 hypomorphs are able to survive past gastrulation (Table 1).

Growth retardation or delay of most hypomorphs was apparent at earlier embryonic stages by smaller body size and reduced somite number compared with WT siblings (Figure 3d); however, a size difference was typically not discernable at later embryonic stages (E14.5-17.5). Neural tube closure defects were observed in $25 \%$ of hypomorphs from E12.5 to E17.5 ( $n=58)$. The main types of neural tube closure defects observed were anterior neural tube defects, including exencephaly. The appearance of neural tube closure defects did not correlate with the appearance of laterality defects.

Because of the increased survival of Zic3 hypomorphs as compared with that of Zic3 nulls (21), a detailed analysis of laterality and cardiac defects was performed. Laterality defects were observed at early embryonic stages by left-sided marker analysis (Figure 4) or heart looping abnormalities (Figure 5 and Table 2) and at later embryonic or fetal stages by complex heart defects (Figure 6 and Table 3 ) and abnormal patterning or positioning of organs as discussed in detail in the following sections. Normal arrangement (situs solitus), abnormal arrangement (situs ambiguus), and right isomerism of organs were observed in hypomorphs at E17.5. Six litters were analyzed at E17.5, containing a total of 18 Zic3 hypomorphs. Of these, eight exhibited normal laterality (situs solitus), three exhibited right isomerism, and seven exhibited situs ambiguus. Right isomerism was identified by bilateral, multilobed lungs
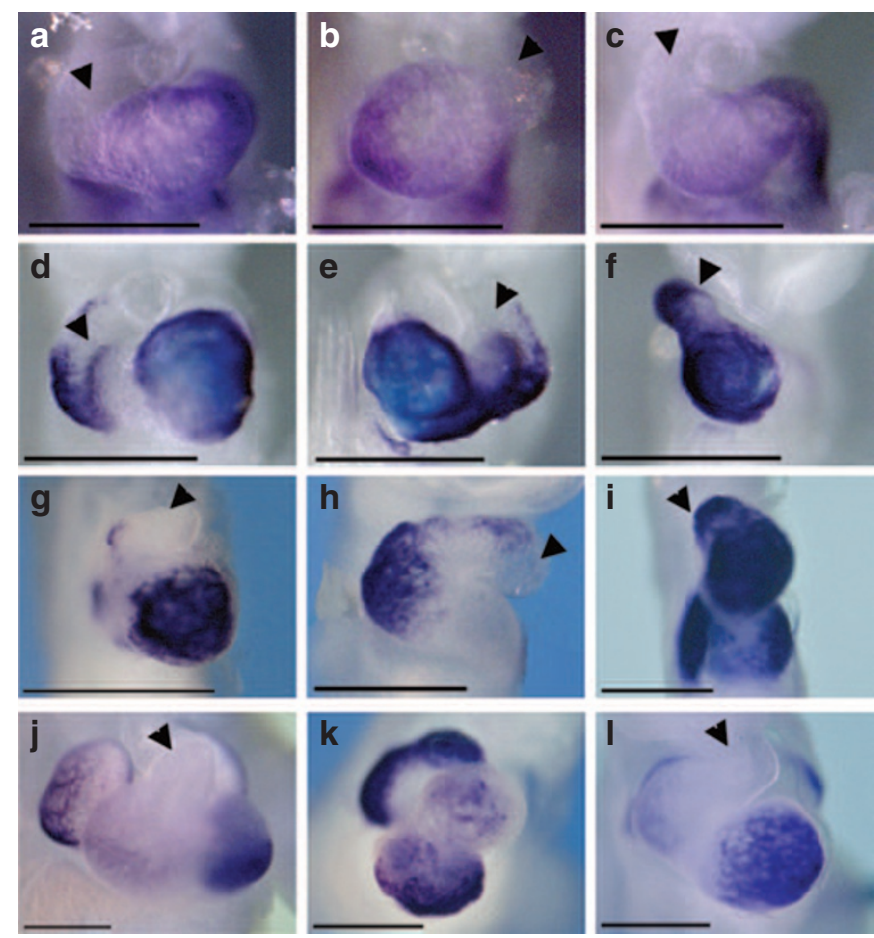

Figure 5. Markers of cardiac differentiation are expressed normally, despite complex looping defects. (a-i) E9.5 embryos and (j-I) E11.5 embryos were analyzed for markers of cardiac differentiation, (a-c) Tbx5 and (d-I) Nppa. Both markers were expressed in all Zic3 null and hypomorphic embryos analyzed, and the level of expression was comparable with wild-type expression levels. Tbx5 was normally localized to the proper morphological location in (b,c) Zic3 null embryos, despite looping abnormalities. See Tbx5 in (a) wild-type embryo for comparison. (d-I) Nppa was normally localized to the proper morphological location in (e,f) Zic3 null and (h,i,k,l) hypomorphic embryos, despite looping abnormalities. For comparison, see Nppa in wild-type embryos at (d) E9.5 and (j) E11.5. Arrowheads, outflow tract. Scale bars, $\times 6$.

(Figure 3j) and a small or absent spleen. Stomach positioning was random in embryos with right isomerism (Table 3). Situs ambiguus was identified by abnormal lung lobation and/ or malpositioning of at least one visceral organ.

\section{Left-Right Marker Analysis in Zic3 Hypomorphs}

Nodal and Pitx2 markers were analyzed by WISH to identify abnormalities in conserved left-right signaling pathways. Nodal is expressed at the head-fold stages symmetrically in perinodal crown cells. Later, Nodal expression is asymmetrical, with increased expression on the left side of the node. At the four- to six-somite stages, Nodal is also expressed in the left lateral plate mesoderm (LPM). Pitx2 is first expressed in the left LPM around the same time as Nodal, but its expression persists much later. Embryos were collected from E7.75 to E9.5 to attain comparable numbers and stages of WTs and Zic3 hypomorphs for left-right marker analysis by Nodal and Pitx2 WISH. Embryos that were early head fold to six-somite stages were used for Nodal WISH, and embryos that were at the 6- to 12-somite stages were used for Pitx2 WISH.

Perinodal Nodal staining was present in six and absent in one of the seven Zic3 hypomorphs at early head-fold stage, 
with robust staining in the six embryos showing staining. Perinodal Nodal staining was present in 11 of 17 and absent in 6 of $17(35 \%)$ one- to three-somite-stage embryos, with clearly attenuated staining in 10 of 11 embryos with staining present. On the contrary, robust Nodal staining was present around the nodes of all 16 WT embryos at early head-fold to three-somite stages. At four- to six-somite stages, only one Zic3 hypomorph exhibited bilateral Nodal expression around the node, and the remaining 17 embryos exhibited no Nodal expression around the node (Figure $4 \mathbf{b}, \mathbf{g}$ ). Of the 13 WT embryos analyzed at these stages, 10 exhibited greater expression of Nodal on the left side of the node; two exhibited symmetrical, bilateral expression; and only one exhibited no expression (Figure 4g). These results were similar to those previously described in Zic3 nulls and heterozygotes, in which Nodal expression was normally initiated around the node but often failed to persist past the two-somite stage (11). These results indicate that there is a threshold requirement for Zic3 expression to maintain Nodal expression at the node. At these same stages, Nodal was expressed in the left LPM of 11 of 13 WT embryos analyzed and absent in the remaining two embryos (Figure 4h). However, Nodal expression was absent in the LPM of 11 of $18 \mathrm{Zic3}$ hypomorphs and present in the left LPM of only 7 hypomorphs (Figure $4 \mathbf{h}$ ). In contrast to previous results in Zic3 null embryos in which Nodal expression was found in bilateral LPM or right LPM, these patterns of misexpression were never identified in Zic3 hypomorphs at the same stage $(n=18)$. Pitx2 was expressed in the left LPM of all 29 WT embryos and in 24 of 45 Zic3 hypomorphic embryos analyzed. Of the remaining hypomorphs, $47 \%$ had abnormal Pitx2 expression patterns: 14\% had bilateral LPM expression, $3 \%$ had right-sided LPM expression, and $4 \%$ had absent LPM expression (Figure 4i).

\section{Cardiovascular Malformations in Zic3 Hypomorphs}

Human heterotaxy demonstrates widely variable cardiac phenotypic presentations, and it was therefore of interest to determine the spectrum of defects identified in these mice with a uniform genetic etiology. Cardiovascular malformations were observed from E8.5 to E17.5. At E9 to E9.5, the most common malformations observed grossly were looping defects, such as leftward (L-) looping or incomplete rightward (D-) looping, or failure of the heart tube to begin differentiating into chambers (Table 2). By E10.5 to E11.5, the malformations observed were more complex, and many hearts contained multiple malformations, the most common being inferior/superior ventricular arrangement, the appearance of a single ventricle, and L looping (Table 2). Pericardial edema was also observed in several embryos at these stages, indicating cardiac failure. Malformations observed at E12.5-14.5 were similar to those at E10.5-11.5, but by this stage, the formation of a single atrium was also observed in some embryos (Table 2).

Table 2. Reduction in Zic3 expression results in an array of heart defects

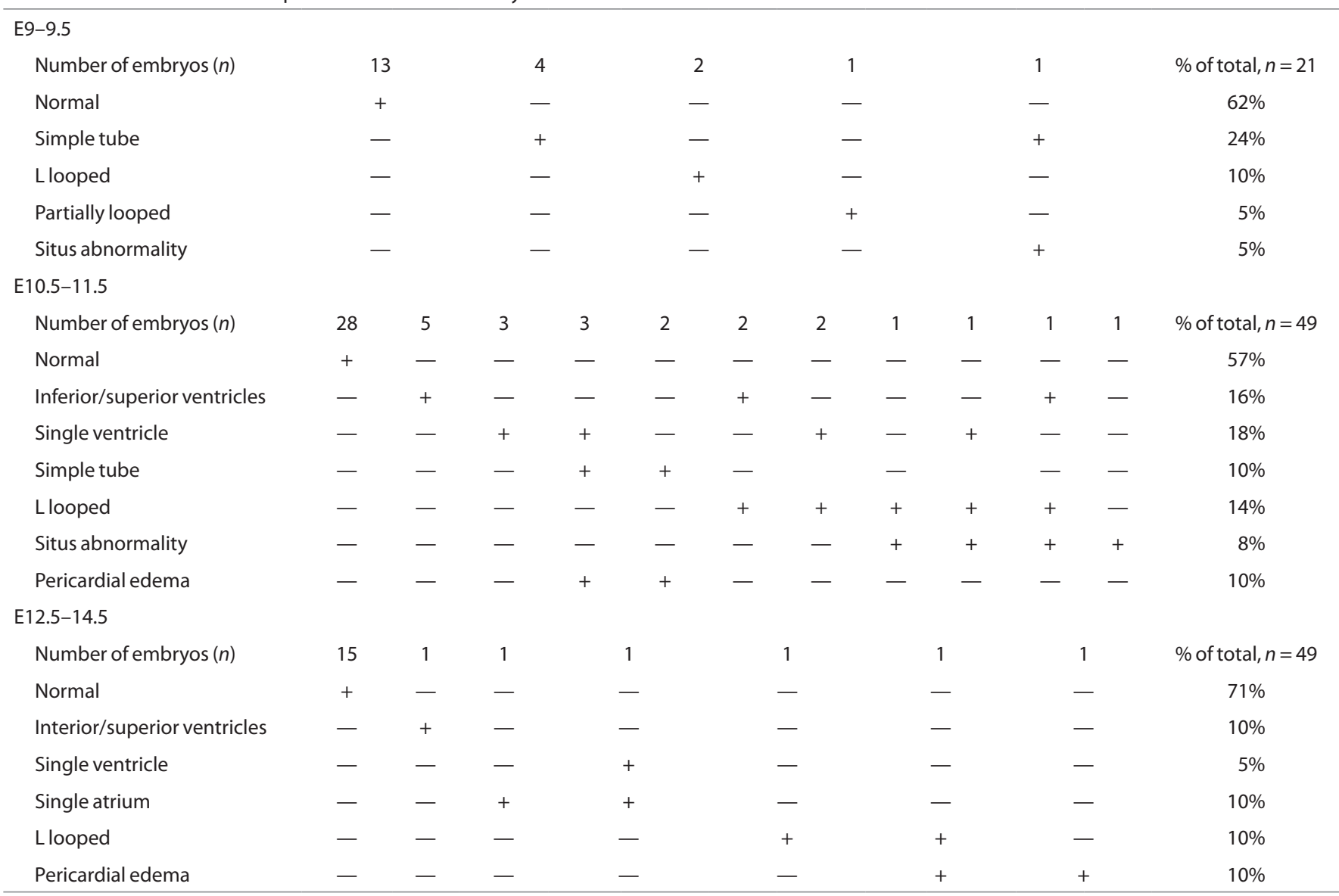


Zic3 has been previously implicated in having a direct role in cardiac development separate from its role in left-right development (20). It was reported that Zic3-null mice have severely reduced expression of atrial natriuretic factor (encoded by $N p p a$ ) and reduced trabecular myocardium. Furthermore, it was reported that $Z i c 3$ null embryonic stem cells have reduced expression of cardiac-specific genes $N p p a, N k \times 2.5$, and Tbx5, compared with WT embryonic stem cells (20). In contrast, in Zic3 null or hypomorphic embryos ( $n=4$ for each gene), analysis of Nppa and Tbx5 gene expression by WISH at E9.5 in our study revealed expression levels that were qualitatively comparable with those in WT embryos (Figure 5). Furthermore, analysis of hearts at E17.5 in Zic3 hypomorphs revealed normal trabecular myocardium compared with that in WT (Figure 6).

In addition to being expressed at relatively normal levels, $N p p a$ and $T b x 5$ also marked the proper morphological locations in the heart regardless of looping abnormalities. Tbx5 is normally expressed in the left ventricle, and it was expressed in the morphological left ventricle of an L-looped heart (Figure $5 \mathrm{~b}$ ) and along the posterior edge of the ventricle in hearts with single ventricles (Figure $5 \mathrm{c}$ ), which is the same expression pattern seen with other left ventricular markers, such as Nppa and Hand1, in single ventricle-containing hearts $(12,24,25)$. Nppa is expressed in the outflow tract, left ventricle, and atria at E9.5; and it was expressed in the proper morphological locations in Zic3 nulls (Figure 5e,f). The hearts of Zic3 hypomorphs were also analyzed by Nppa WISH, and expression was seen in the proper morphological locations with levels comparable with WTs. Nppa localization was useful for identifying the region specified as left ventricle in tubular hearts without chamber formation at E9.5 (Figure 5h) and for identifying specific chambers in hearts with very complex looping abnormalities at E11.5 (Figure 5k).

At E17.5 18 of 46 embryos were Zic3 hypomorphs (39\%), of which 10 had morphological heart defects (56\%) (Table 3 ). The remaining eight hypomorphs had normal organ asymmetry, heart morphology, and proper levocardial positioning in the chest comparable with WT controls (Figure 6a-f). Two hypomorphs with pulmonary and abdominal reversal had hearts with dextrocardial positioning in the chest and anteriorly positioned aortas (Figure $6 \mathrm{~g}-\mathbf{i}$ ). Sectioning of hearts revealed double-outlet right ventricle with subaortic ventricular septal defects (Figure 6j-1). The three hypomorphs with right isomerism sequence had hearts with mesocardial positioning in the chest, right atrial isomerism, and TGA (Figure $6 \mathrm{~m}-\mathbf{0}$ ). Sectioning of hearts revealed bilateral symmetrical venous valves and atrioventricular septal defects (Figure 6p-r). The four remaining hypomorphs had variable heart phenotypes, as described in Table 3.

\section{DISCUSSION}

Heterotaxy is the most highly heritable heart defect (26). Although the genetic causes are not identified in the majority of patients with heterotaxy, mutations in the zinc finger transcription factor ZIC3 are the known cause of the X-linked form of heterotaxy. The spectrum of cardiovascular malformations

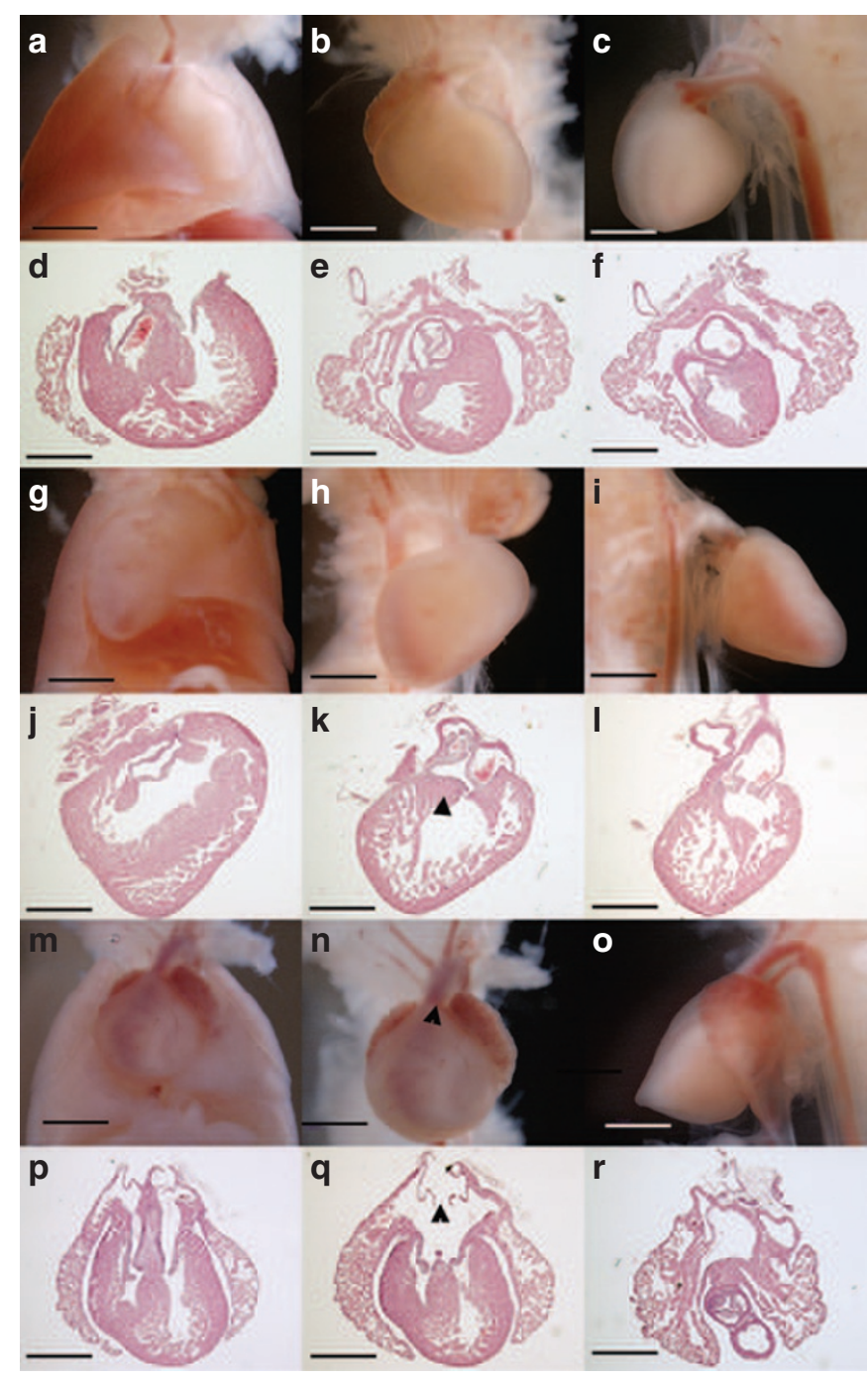

Figure 6. Complex cardiovascular malformations in E17.5 Zic3 hypomorphs. Gross and histologic transverse sections from wild-type (a-f) E17.5 embryos and (g-r) Zic3 hypomorphs. Wild-type embryos have levocardial positioning in chest ((a) frontal view) and proper orientation of chambers and great arteries $((\mathbf{b}, \mathbf{c})$ frontal and lateral views and $(\mathbf{d}-\mathbf{f})$ transverse sections). (g-l) A representative Zic3 hypomorphic embryo demonstrates dextrocardial positioning in $(\mathbf{g})$ the chest with right aortic arch ((h,i) frontal and lateral views) and (j-I) DORV with subaortic VSD ((k) triangle). Another Zic3 hypomorphic embryo had mesocardial positioning in the chest (m), atrial isomerism, TGA, and an anteriorly positioned aorta ((n) triangle; $(\mathbf{o}))$. Transverse sections demonstrate these defects as well as AVSD (p-r) and symmetrical venous valves ((q) triangle). All sections are in transverse plane, shown posterior to anterior. Scale bars, original magnification $\times 2$. AVSD, atrioventricular septal defect; DORV, double-outlet right ventricle; TGA, transposition of the great arteries; VSD, ventricular septal defect.

identified in patients with heterotaxy, including those with ZIC3 mutations, is broad and an understanding of the mechanistic basis for this range of developmental defects is lacking. In this article, we identify a new hypomorphic Zic3 mouse created by targeted insertion of a neomycin cassette and demonstrate the use of this model for investigating molecular and pathologic effects of decreased Zic3 expression. In contrast to recently published work, our data do not support a novel role 
Table 3. Phenotypes of E17.5 Zic3 hypomorphs from six litters

\begin{tabular}{|c|c|c|}
\hline $\begin{array}{l}\text { Number of } \\
\text { embryos }\end{array}$ & Overall phenotype & Cardiac phenotype \\
\hline 7 & Normal (situs solitus) & Normal (levocardia) \\
\hline 1 & Right pulmonary isomerism; asplenia, stomach on left & $\begin{array}{l}\text { Mesocardia, TGA, right atrial isomerism, AVSD, } \\
\text { and symmetrical venous valves }\end{array}$ \\
\hline 1 & $\begin{array}{l}\text { Right pulmonary isomerism; asplenia; stomach on right; intestines } \\
\text { malpositioned outside of liver; anal atresia }\end{array}$ & $\begin{array}{l}\text { Mesocardia, TGA, right atrial isomerism, AVSD } \\
\text { and symmetrical venous valves }\end{array}$ \\
\hline 1 & Right pulmonary isomerism; asplenia; stomach large and centrally located & $\begin{array}{l}\text { Mesocardia, TGA, right atrial isomerism, AVSD, } \\
\text { and symmetrical venous valves }\end{array}$ \\
\hline 1 & Situs ambiguus; lungs abnormally multilobed but not symmetrical & DORV with VSD; normal otherwise (levocardia) \\
\hline 1 & $\begin{array}{l}\text { Situs ambiguus; lungs abnormally multilobed but not symmetrical; } \\
\text { intestinal atresia }\end{array}$ & $\begin{array}{l}\text { Bottom of heart flat; single ventricle; single } \\
\text { atrium (right); only one set of A } / \mathrm{V} \text { valves; right } \\
\text { descending aorta; anteriorly positioned aorta }\end{array}$ \\
\hline 1 & $\begin{array}{l}\text { Situs ambiguus; lungs abnormally multilobed but not symmetrical; severe } \\
\text { exencephaly and anterior neural tube closure defect; microcephaly and } \\
\text { facial malformations (low-set ears, no eyes); rib dysplasia }\end{array}$ & $\begin{array}{l}\text { Large RA, small LA; AVSD; right descending } \\
\text { aorta; TGA }\end{array}$ \\
\hline 1 & $\begin{array}{l}\text { Situs ambiguus; lungs abnormally multilobed but not symmetrical; severe } \\
\text { exencephaly and anterior neural tube closure defect; microcephaly and } \\
\text { facial malformations (low-set ears, no eyes); rib dysplasia }\end{array}$ & AVSD; right descending aorta; TGA \\
\hline 1 & Pulmonary and abdominal situs inversus & $\begin{array}{l}\text { Dextrocardia; anteriorly positioned aorta; } \\
\text { DORV with subaortic VSD }\end{array}$ \\
\hline 1 & Pulmonar and abdominal situs inversus; microphthalmia & $\begin{array}{l}\text { Dextrocardia; anteriorly positioned aorta; } \\
\text { DORV with subaortic VSD }\end{array}$ \\
\hline
\end{tabular}

AVSD, atrioventricular septal defect; DORV, double-outlet right ventricle; LA, left atrium; RA, right atrium; TGA, transposition of the great arteries; VSD, ventricular septal defect.

for Zic3 in the myocardium via regulation of Nppa and Tbx5 and suggest that the primary effect of Zic3 on cardiac development occurs during left-right patterning stages.

It is not uncommon for the insertion of loxP sites and selectable markers to result in hypomorphic alleles, which are instructive about gene function (27-29). Previously, Nodal hypomorphs illustrated the role of Nodal in left-right patterning because they circumvented the gastrulation stage lethality identified in Nodal null mice. Similarly, Zic3 hypomorphs prove to be a more useful model for studying cardiac defects than Zic3 nulls because there is less early lethality resulting from gastrulation defects. Unlike Nodal null mice, which never survive past gastrulation, a subset of Zic3 null mice are able to survive past gastrulation and be born as healthy, fertile adults. There was a higher penetrance of grossly detectable heart defects in Zic3 hypomorphs (38\%, $n=24)$ at E12.5 compared with those previously described in Zic3 nulls (14). Furthermore, at E17.5, the penetrance of cardiovascular defects was even higher in Zic3 hypomorphs (56\%, $n=18$, six litters) than that seen grossly at E12.5 and was associated with increasing complexity of the heart and remodeling of the great arteries. The higher penetrance in hypomorphs at later vs. earlier stages may, in part, result from more sensitive detection based on full histological analysis in all fetuses. On the contrary, very few Zic3 null embryos survive to these later stages, and few survivors have morphologically abnormal hearts (14). Thus, the Zic3 hypomorphic mice represent a useful model to better understand the developmental basis of the variable cardiac phenotypes identified in heterotaxy disorders.

Analyses of molecular markers of left-right patterning in Zic3 hypomorphs demonstrate some important differences as compared with Zic3 null embryos. In Zic3 hypomorphs, a higher percentage of embryos have normal left-sided Nodal expression in the left LPM than in Zic3 nulls (40\% vs. 20\%). In addition, Nodal is never identified in the right LPM or bilaterally in Zic3 hypomorphs despite analysis of nearly fourfold more embryos than Zic3 nulls at the same stages. This partial rescue of left-right signaling by low levels of Zic3 expression in the hypomorphs suggests a threshold or dose-related response of Nodal in the LPM to Zic3.

When comparing early left-right patterning molecular marker abnormalities with later anatomic abnormalities, we note that there is strong concordance between abnormal Pitx2 expression at the $6-12$-somite stage $(53 \%, n=45)$ and abnormal anatomy at E17.5 $(56 \%, n=32)$. These results suggest that if Zic3 has a later role in cardiac development separate from its role in left-right patterning, it accounts for a minority of the identified abnormalities.

Anatomic differences were noted between Zic3 hypomorphs and Zic3 null mice at fetal stages analogous to the results seen with earlier stage molecular markers. Left pulmonary isomerism was not observed in any hypomorph, whereas Zic3 nulls exhibit both right and left pulmonary isomerism and 
pulmonary reversal (11). Because the lungs are the only organs to appear asymmetric at the earliest stages of their development (30), it is possible that bilateral misexpression of Nodal, and not Pitx2, in the LPM causes left pulmonary isomerism. Indeed, in different mouse models exhibiting left pulmonary isomerism, Nodal is misexpressed bilaterally in the LPM (31-33).

To further address a possible role for Zic3 in the cardiac compartment, cardiac expression was analyzed by WISH or in multiple Zic3-LacZ-BAC transgenic mouse lines, but no detectable gene expression was identified. These data, combined with the normal expression of cardiac markers, Tbx5 and Nppa, normal myocardial trabeculation at later stages, and concordance of the penetrance of left-right marker molecular abnormalities and later stage organ abnormalities suggest that heart defects in Zic3null and hypomorphic mice are secondary to left-right patterning defects. This has been further supported by two recent studies using conditional approaches to eliminate Zic3 expression in the cardiac compartment $(34,35)$. Zic3 hypomorphic mice are useful models for analyzing laterality-spectrum heart defects, and they will be used in future studies to further investigate the etiology of heart defects associated with Zic3 loss of function.

\section{METHODS}

\section{Gene Targeting}

An $\sim 12-\mathrm{kb}$ region was subcloned from a 129 BAC clone that included the Zic3 locus, and a pGKNeo cassette was incorporated so that a single loxP site was inserted upstream of Zic3 exon 1 and a loxP/FRTflanked Neo cassette was inserted downstream of exon 1 in the first intron (Figure 1a). The targeting vector was confirmed by sequencing and then electroporated into C57BL6/129 embryonic stem cells for gene targeting by homologous recombination. Properly targeted embryonic stem cells were injected into C57BL6 blastocysts, and resulting chimeras were bred with C57BL6 mice to achieve germline transmission, which was verified by Southern blot analysis (data not shown). Floxed mice carrying the Neo cassette within intron 1 are referred to as $\mathrm{Zic}^{\mathrm{NeoIn} 1 / \mathrm{WT}}$.

\section{Embryo Collection and Genotyping}

All animal experiments conformed with the Guide for the Care and Use of Laboratory Animals published by the US National Institutes of Health and were approved by the Institutional Animal Care and Use Committee of Cincinnati Children's Hospital Medical Center.

Mice were euthanized by $\mathrm{CO}_{2}$ inhalation, followed by cervical dislocation. E7.5 to 17.5 mouse embryos were collected following timed matings of $Z i c 3^{\text {NeoIn1/WT }}$ females with $Z i c 3^{\text {NeoIn1/y }}$ males $\left(Z i c 3^{\text {NeoIn1/NeoIn1 }}\right.$ females are infertile). Because Zic3 hypomorphs are often delayed compared with WT siblings, presomitic stages were matched by Theiler criteria and postsomitic stages were matched by somite number. Embryos were genotyped using primers to detect sex-determining factor on chromosome $Y(11)$ and the following primers that differentiate between WT and hypomorphic Zic3 alleles: SDL1: 5'-CGT TCT CAA GGT GGT GAG GCA GCA G-3' and SDL2: 5'-GAA AGG GAT CCG CCG GGT TTG CG-3’.

\section{Embryo Processing}

Embryos were fixed in $4 \%$ paraformaldehyde/phosphate-buffered saline (Electron Microscopy Sciences, Hatfield, PA) before WISH and $\beta$-galactosidase detection; the latter was performed as previously described (14).

The organ arrangement and external heart phenotype was observed in E17.5 hypomorphs before the heart was removed for embedding. Before embedding, hearts were fixed in $4 \%$ paraformaldehyde/phosphate-buffered saline and dehydrated in ethanol. Hearts were then washed with xylene for $15 \mathrm{~min}, 1: 1$ xylene/paraffin wax for $20 \mathrm{~min}$, and then three times in paraffin wax for $1 \mathrm{~h}$ per wash. Hearts were embedded in paraffin wax with the apex of the heart oriented toward the top of the block. Hearts were cut transversely into $12 \mu \mathrm{m}$ sections from apex to aortic arch (posterior to anterior). Sections were stained with hematoxylin and eosin.

\section{Analysis of Gene Expression}

WISH was performed as described previously (11). For real-time RT-PCR, whole E12.5 embryos were collected in RNAlater (Ambion, Austin, TX). RNA extractions were performed using a Totally RNA kit (Ambion). cDNA was generated using a High-Capacity cDNA Reverse Transcription Kit (Applied Biosystems, Foster City, CA). Real-time PCR was performed using an ABI PRISM 7000 Sequence Detection System (Applied Biosystems) and Power SYBR Green PCR Master Mix (Applied Biosystems), with intron spanning primer pairs. Zic3 gene expression results were normalized to GAPDH. The following primers were used: GAPDH-F: 5'-TGCGACTTCAACAGCAACTC and GAPDH-R: 5'-GCCTCTCTTGCTCAGTGTCC; Zic3-F1: 5'-CGGGCTGCGGGAAGAT and Zic3-R1: 5'-CTCACCTGTAT GGGTCCTCTTGT; Zic3-F2: 5'-CCCTGCGCAAACACATGA and Zic3-R2: 5'-GGGAGGAATCTGACCCTTGAG.

Two sets of primer pairs for Zic3, flanking intron 1 (Zic3 F1/Zic3 R1) and intron 2 (Zic3 F2/Zic3 R2), were used independently for double verification of the Zic3 expression results. Six independent reactions were run for each biological sample with each of the three primer pairs. Histograms represent relative expression \pm SE.

\section{ACKNOWLEDGMENTS}

We thank B. Bruneau for Nppa and Tbx5 in situ probes. We also thank R.B. Hinton for helpful discussions on cardiac malformations in heterotaxy.

\section{STATEMENT OF FINANCIAL SUPPORT}

This work was supported by the National Institutes of Health (grants R01 HL088639 and R01 HL088639-03S1 to S.M.W.).

\section{Disclosure: The authors declare no conflict of interest.}

\section{REFERENCES}

1. Gebbia M, Ferrero GB, Pilia G, et al. X-linked situs abnormalities result from mutations in ZIC3. Nat Genet 1997;17:305-8.

2. Sutherland MJ, Ware SM. Disorders of left-right asymmetry: heterotaxy and situs inversus. Am J Med Genet C Semin Med Genet 2009;151C:30717.

3. Ware SM, Peng J, Zhu L, et al. Identification and functional analysis of ZIC3 mutations in heterotaxy and related congenital heart defects. Am J Hum Genet 2004;74:93-105.

4. Aruga J, Nagai T, Tokuyama T, et al. The mouse zic gene family. Homologues of the Drosophila pair-rule gene odd-paired. J Biol Chem 1996;271:1043-7.

5. Bedard JE, Haaning AM, Ware SM. Identification of a novel ZIC3 isoform and mutation screening in patients with heterotaxy and congenital heart disease. PLoS ONE 2011;6:e23755.

6. Mizugishi K, Aruga J, Nakata K, Mikoshiba K. Molecular properties of Zic proteins as transcriptional regulators and their relationship to GLI proteins. J Biol Chem 2001;276:2180-8.

7. Aruga J, Yokota N, Hashimoto M, Furuichi T, Fukuda M, Mikoshiba K. A novel zinc finger protein, zic, is involved in neurogenesis, especially in the cell lineage of cerebellar granule cells. J Neurochem 1994;63:1880-90.

8. Zhu J, Nakamura E, Nguyen MT, Bao X, Akiyama H, Mackem S. Uncoupling Sonic hedgehog control of pattern and expansion of the developing limb bud. Dev Cell 2008;14:624-32.

9. Quinn ME, Haaning A, Ware SM. Preaxial polydactyly caused by Gli3 haploinsufficiency is rescued by Zic3 loss of function in mice. Hum Mol Genet 2012;21:1888-96.

10. Kitaguchi T, Nagai T, Nakata K, Aruga J, Mikoshiba K. Zic3 is involved in the left-right specification of the Xenopus embryo. Development 2000;127:4787-95.

11. Purandare SM, Ware SM, Kwan KM, et al. A complex syndrome of leftright axis, central nervous system and axial skeleton defects in Zic3 mutant mice. Development 2002;129:2293-302. 
12. Ware SM, Harutyunyan KG, Belmont JW. Heart defects in X-linked heterotaxy: evidence for a genetic interaction of $\mathrm{Zic} 3$ with the nodal signaling pathway. Dev Dyn 2006;235:1631-7.

13. Carrel T, Purandare SM, Harrison W, et al. The X-linked mouse mutation Bent tail is associated with a deletion of the Zic3 locus. Hum Mol Genet 2000;9:1937-42.

14. Czosek RJ, Haaning A, Ware SM. A mouse model of conduction system patterning abnormalities in heterotaxy syndrome. Pediatr Res 2010;68:275-80.

15. Cast AE, Gao C, Amack JD, Ware SM. An essential and highly conserved role for Zic3 in left-right patterning, gastrulation and convergent extension morphogenesis. Dev Biol 2012;364:22-31.

16. Chhin B, Hatayama M, Bozon D, et al. Elucidation of penetrance variability of a ZIC3 mutation in a family with complex heart defects and functional analysis of ZIC3 mutations in the first zinc finger domain. Hum Mutat 2007;28:563-70.

17. Chung B, Shaffer LG, Keating S, Johnson J, Casey B, Chitayat D. From VACTERL-H to heterotaxy: variable expressivity of ZIC3-related disorders. Am J Med Genet A 2011;155A:1123-8.

18. De Luca A, Sarkozy A, Consoli F, et al. Familial transposition of the great arteries caused by multiple mutations in laterality genes. Heart 2010;96:673-7.

19. Mégarbané A, Salem N, Stephan E, et al. X-linked transposition of the great arteries and incomplete penetrance among males with a nonsense mutation in ZIC3. Eur J Hum Genet 2000;8:704-8.

20. Zhu L, Harutyunyan KG, Peng JL, Wang J, Schwartz RJ, Belmont JW. Identification of a novel role of ZIC3 in regulating cardiac development. Hum Mol Genet 2007;16:1649-60.

21. Ware SM, Harutyunyan KG, Belmont JW. Zic3 is critical for early embryonic patterning during gastrulation. Dev Dyn 2006;235:776-85.

22. Hashimoto K, Nakatsuji N. Formation of the primitive streak and mesoderm cells in mouse embryos - detailed scanning electron microscopical study. Dev Growth Differ 1989;31:209-218.

23. Tam PP, Behringer RR. Mouse gastrulation: the formation of a mammalian body plan. Mech Dev 1997;68:3-25.
24. Takeuchi JK, Ohgi M, Koshiba-Takeuchi K, et al. Tbx5 specifies the left/ right ventricles and ventricular septum position during cardiogenesis. Development 2003;130:5953-64.

25. Koshiba-Takeuchi K, Mori AD, Kaynak BL, et al. Reptilian heart development and the molecular basis of cardiac chamber evolution. Nature 2009;461:95-8.

26. Øyen N, Poulsen G, Boyd HA, Wohlfahrt J, Jensen PK, Melbye M. Recurrence of congenital heart defects in families. Circulation 2009;120:295301.

27. Meyers EN, Lewandoski M, Martin GR. An Fgf8 mutant allelic series generated by Cre- and Flp-mediated recombination. Nat Genet 1998;18:13641.

28. Lowe LA, Yamada S, Kuehn MR. Genetic dissection of nodal function in patterning the mouse embryo. Development 2001;128: 1831-43.

29. Saijoh Y, Oki S, Ohishi S, Hamada H. Left-right patterning of the mouse lateral plate requires nodal produced in the node. Dev Biol 2003;256:16072.

30. Warburton D, El-Hashash A, Carraro G, et al. Lung organogenesis. Curr Top Dev Biol 2010;90:73-158.

31. Constam DB, Robertson EJ. SPC4/PACE4 regulates a TGFbeta signaling network during axis formation. Genes Dev 2000;14:1146-55.

32. Meno C, Shimono A, Saijoh Y, et al. lefty-1 is required for left-right determination as a regulator of lefty-2 and nodal. Cell 1998;94: 287-97.

33. Tsukui T, Capdevila J, Tamura K, et al. Multiple left-right asymmetry defects in Shh(-/-) mutant mice unveil a convergence of the shh and retinoic acid pathways in the control of Lefty-1. Proc Natl Acad Sci USA 1999;96:11376-81.

34. Jiang Z, Zhu L, Hu L, et al. Zic3 is required in the extra-cardiac perinodal region of the lateral plate mesoderm for left-right patterning and heart development. Hum Mol Genet 2013;22:879-89.

35. Sutherland MJ, Wang S, Quinn ME, Haaning A, Ware SM. Zic3 is required in the migrating primitive streak for node morphogenesis and left-right patterning. Hum Mol Genet 2013;22:1913-23. 